\title{
A MODEL OF ION TRANSPORT IN CONJUGATED POLYMERS
}

\author{
JOHN BURNELL ${ }^{1}$
}

(Received 19 April, 2009; revised 31 April, 2009)

\begin{abstract}
Understanding ion transport in conjugated polymers is essential for developing mathematical models of applications of these materials. Previous experimental studies have suggested that cation transport in a conjugated polymer could be either diffusion or drift controlled, with debate over which dominates. In this paper we present a new model of cation transport that explains most of the features seen in a set of recent experiments. This model gives good agreement with measured concentration profiles, except when the profile has penetrated the polymer by more than $60 \%$. The model shows that both diffusion and drift processes can be present. An application of a micro-actuator based on a conjugated polymer is presented to demonstrate that this technology could be used to develop a micro-pump.
\end{abstract}

2000 Mathematics subject classification: primary 76R99; secondary 35Q80.

Keywords and phrases: ion transport, conjugated polymers.

\section{Introduction}

Conjugated polymers are materials with potential applications in a large number of areas. Many of these applications are based on controlling the properties of the polymer through electrochemical processes. In addition to utilizing the conductive properties, many potential applications in the biomedical area arise from using the ability of the polymer to change volume [2]. The advantages of using a conjugated polymer for these applications is the lack of mechanical parts together with the ability to create micron-scale or smaller components.

A key feature of the electrochemical process in conjugated polymers is the transport of ions from the electrolyte into and out of the conducting polymer. There is currently considerable debate about the key mechanisms influencing the transport of these ions [4]. Both advective and diffusive mechanisms have been suggested as the key processes. The first part of this paper introduces a model which combines both these processes.

\footnotetext{
${ }^{1}$ Industrial Research Limited, Lower Hutt, New Zealand; e-mail: j.burnell@irl.cri.nz.

(C) Australian Mathematical Society 2009, Serial-fee code 1446-1811/2009\$16.00
} 


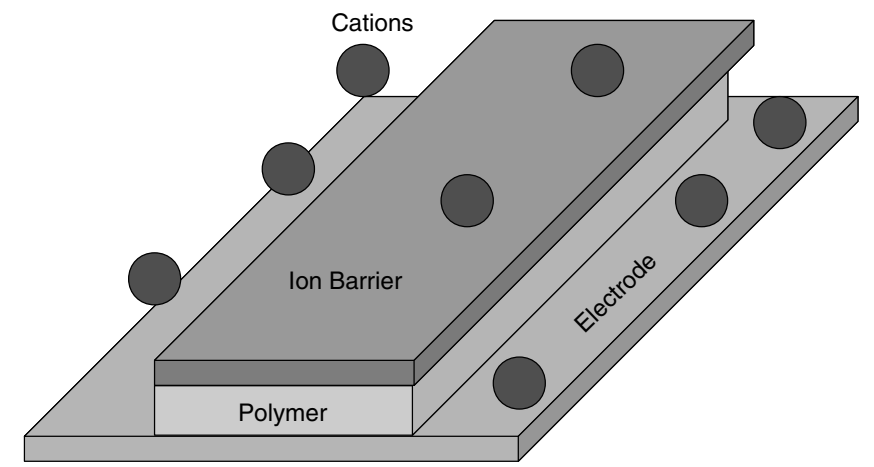

FIgURE 1. Model configuration corresponding to experiments of Wang et al. [4]. A thin strip of conjugated polymer was placed on an electrode and surrounded by an electrolyte. A transparent film which acts as a barrier to ion transport was placed on the upper surface of the polymer. Ion transport into the polymer then occurs laterally through the sides of the polymer.

When a reducing potential is applied to a conjugated polymer, electrons are transferred to the polymer backbone and an excess of negative charge builds up in the polymer. If an electrolytic solution surrounds the polymer then cations are drawn into the polymer. During this reduction phase, solvent molecules accompany the cations transported into the polymer. Plieth et al. [1] estimated that three water molecules were carried with each $\mathrm{K}^{+}$ion in their experiments. The volume occupied by the cation and accompanying solvent molecules typically gives rise to a volume increase of the polymer. This volume increase can be exploited to make a micro-actuator.

The literature describes experiments that show that the transport of cations in a conjugated polymer is diffusive or is driven by a drift model. Different authors have seen evidence for each process. Wang et al. [4] performed experiments to investigate the importance of each process. Their results suggest that both processes are present, but typically one of them is dominant. These experiments suggest that the magnitude of the applied potential controls which process is dominant.

The conjugated polymer used in [4] was polypyrrole doped with dodecylbenzenesulfonate, PPy(DBS). This choice of doping ion, DBS, is important as it is sufficiently large that it remains in the polymer during the reduction and oxidation (redox) reactions. This means that transport is only occurring through the cationsolvent complexes together with the electrons.

The experiments of Wang et al. [4] were designed using a thin strip of a conjugated polymer material placed on an electrode and covered on the top face with an ionblocking layer. This arrangement means that ion transport into the polymer can only take place through the edges. Further, the thinness of the polymer means that the distance that the electrons travel is small compared to the width of the polymer strip. So the rate limiting process in the experiment is the lateral transport of cations. This arrangement is illustrated in Figure 1.

A further feature of the experiments is that when the polymer is reduced from a fully oxidized state, the material undergoes a colour change. By tracking this colour 
change it possible to see the front between the oxidized and reduced material. By interpreting the colour as representing an ion concentration, profiles of concentration across the polymer were produced.

For small reduction potentials there was no clearly defined front, suggesting that diffusive processes were dominant. As the size of the potential was increased, a sharp phase front was observed which moved with a constant velocity for a certain time. This behaviour suggests that an advective, or drift, process is dominating in this regime.

Wang et al. [4] developed a simple model of cation transport corresponding to these experiments. This model gave shapes of the concentration profiles broadly consistent with the profiles inferred from the experiments, but the velocities predicted by the model did not agree with the experimental values. The purpose of this paper is firstly to investigate a model which more accurately matches the cation concentration profiles, and secondly to describe an application of the volume change associated with the redox reactions to develop a micro-pump.

\section{The model}

The model proposed by Wang et al. [4] is a first approximation to the transport processes that occur when a reducing potential is applied to an oxidized polymer. The equations describing the model are:

$$
\frac{\partial C}{\partial t}=-\nabla \cdot(-D \nabla C-z \mu \nabla \phi), \quad \epsilon_{0} \nabla(\epsilon \nabla \phi)=C-n,
$$

where $C$ is the cation concentration $\left(\mathrm{mol} \mathrm{m}^{-3}\right), D$ is a diffusion coefficient $\left(\mathrm{m}^{2} \mathrm{~s}^{-1}\right)$, $z$ is the charge of the ion and $\mu$ is the mobility $\left(\mathrm{m}^{2}(\mathrm{Vs})^{-1}\right), \epsilon_{0}$ is the permittivity, $\epsilon$ the dielectric constant of the polymer and $n$ is the electronic charge density. These equations apply in the spatial region occupied by the polymer. It is assumed that this region does not evolve with time-that is, the effects of the volume change in the polymer are ignored in this model.

The first of the equations in (2.1) describes mass conservation of the ions, where the transport term, the right-hand side, is composed of a diffusive and a drift term. The diffusive term accounts for ion transport driven by the concentration gradient, and the drift term is due to the electric field resulting from the applied potential. The second equation describes this potential in terms of the net charge density, $C-n$, following from Maxwell's equations.

When applying this system to the experiments of Wang et al., the region can be considered a slab, as shown in Figure 1. The polymer strip is $0.3 \mu \mathrm{m}$ high, $300 \mu \mathrm{m}$ wide and $8000 \mu \mathrm{m}$ long. Cation transport into the conducting polymer occurs laterally; electron transport is assumed instantaneous in the direction perpendicular to the electrode. If the width of the strip of polymer is denoted by $2 W$ and the electrolyte is assumed to be well stirred then the boundary conditions are

$$
\left.C\right|_{x= \pm W}=C_{0}
$$

where $C_{0}$ is the cation concentration in the electrolyte. 


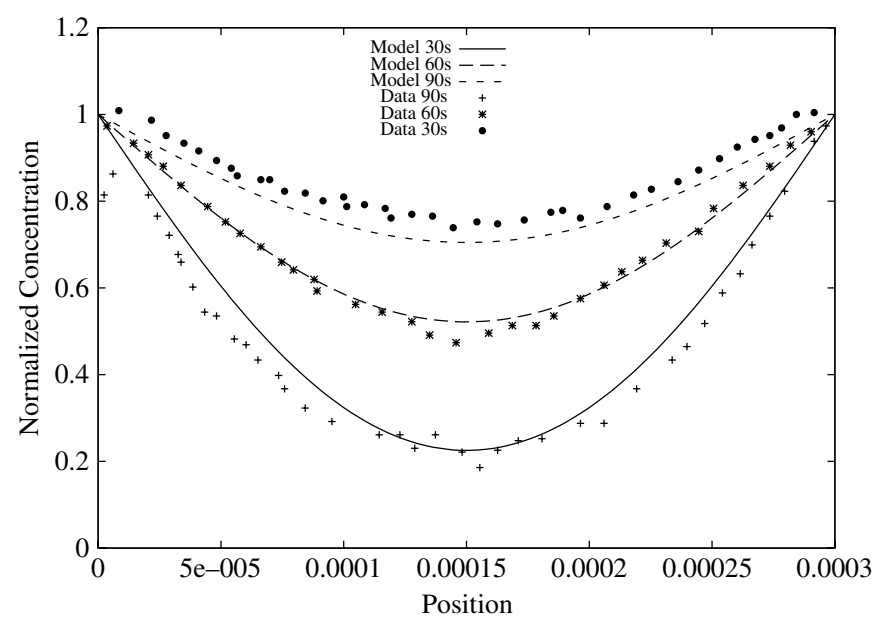

FIGURE 2. Match of the model concentration from (2.1) to the experimental data of Wang et al. [4] for an applied potential of $-0.8 \mathrm{~V}$. In this case the transport of cations shows a diffusive characteristic.

The assumption that the region occupied by the polymer does not change with time is reasonable as growth in the polymer is usually of the order of a few microns compared to the width of the strip of polymer of $300 \mu \mathrm{m}$.

This model was applied to this experimental set-up and solved using a 2D finiteelement method with linear shape functions. The modelled dimensions were the width and length of the polymer. The diffusion coefficient and mobility were adjusted until the results provided a good match to the experimental data. The results of this process are shown in Figures 2 (low applied potential) and 3 (high applied potential). The model provides a good match to the case with a low applied potential where diffusion dominates. For the higher applied potential case, the model provides a reasonable match to the velocity of the concentration fronts up to 1.5 seconds. After 1.5 seconds, the model velocity is too slow. Also the shape of the concentration profiles near the boundaries of the strip is poorly matched. This behaviour suggests that the model of (2.1) is lacking some important processes.

The purpose of this work was to extend the model given in (2.1) to provide a more accurate description of the concentration profiles for high applied potentials. The key extensions are the following.

- To model the state of the cations. It is assumed that as some of the cations move near to the DBS doping ions they will be electrostatically attracted and will therefore be unavailable to move further into the polymer.

- To represent this by assuming that the cations have mobile and immobile states.

- To model the cation concentration in the electrolyte as well as in the polymer.

In the model we identify mobile and immobile ions with concentrations $C^{m}$ and $C^{i}$, respectively. The model equations for this system are 


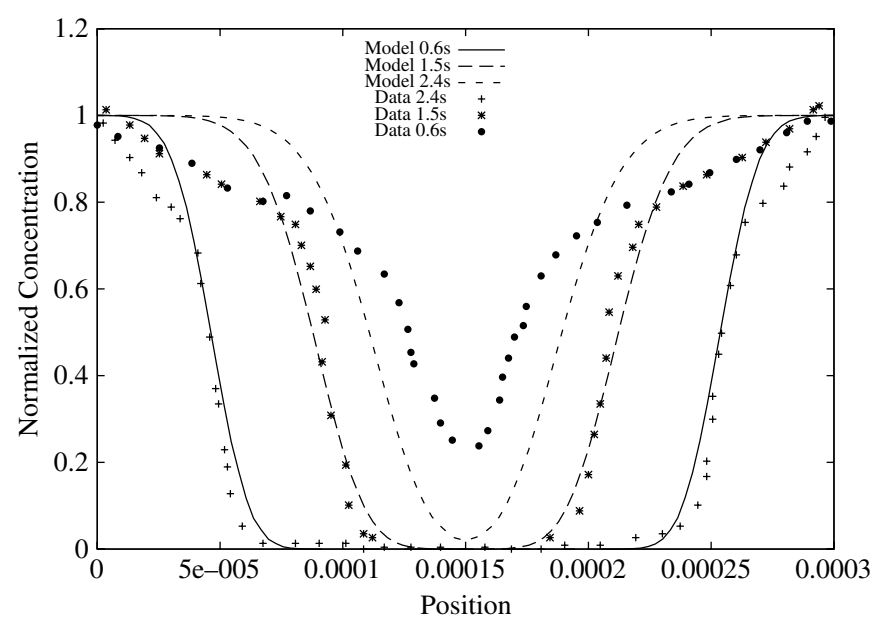

FIGURE 3. Match of the model concentration from (2.1) to the experimental data of Wang et al. [4] for an applied potential of $-1.5 \mathrm{~V}$. In this case the concentration fronts move much faster than shown in the previous case in Figure 2. The shape of the concentration profiles indicates that advective transport of cations is the dominant process.

$$
\begin{gathered}
\frac{\partial C^{m}}{\partial t}=-\nabla \cdot\left(-D^{m} \nabla C^{m}-z \mu \nabla \phi\right)-\alpha C^{m}\left(C_{a}-C^{i}\right) \quad \text { in the polymer, } \\
\frac{\partial C^{m}}{\partial t}=-\nabla \cdot\left(-D^{m} \nabla C^{m}-z \mu \nabla \phi\right) \quad \text { in the electrolyte } \\
\frac{\partial C^{i}}{\partial t}=\alpha C^{m}\left(C_{a}-C^{i}\right), \quad \epsilon_{0} \nabla(\epsilon \nabla \phi)=C^{m}+C^{i}-n
\end{gathered}
$$

where $D^{m}$ is now the diffusion coefficient of the mobile cations; $C_{a}$ is an equilibrium concentration of immobile cations; $\alpha$ is a transfer coefficient $\left(\mathrm{m}^{3} \mathrm{~s}^{-1} \mathrm{~mol}^{-1}\right)$ between the mobile and immobile ions. The other terms are the same as for (2.1).

The key feature of this equation is the transfer term between the mobile and immobile ions $\alpha C^{m}\left(C_{a}-C^{i}\right)$. As the cations enter the polymer they are mobile and can move freely, but as they move through the polymer they come into the vicinity of the large negatively charged DBS ions. The attraction to the negative ion reduces the ability of some of the cations to move and they are deemed immobile. The transfer between mobile and immobile states is modelled as depending on the concentration of mobile ions and the availability of DBS ions. Since the concentration of the DBS ions is not explicitly modelled in this system, a surrogate is the last term in the first equation of (2.2), $C_{a}-C^{i}$. That is, as the concentration of immobile ions tends to an equilibrium value the transfer term reduces to zero.

In addition to including the mobile and immobile states, the problem domain is extended to include transport of the cations in the electrolyte. In the electrolyte it is assumed that all ions are mobile with a different diffusion coefficient than inside the polymer. The lateral boundary conditions are applied at the edge of the electrolyte, 


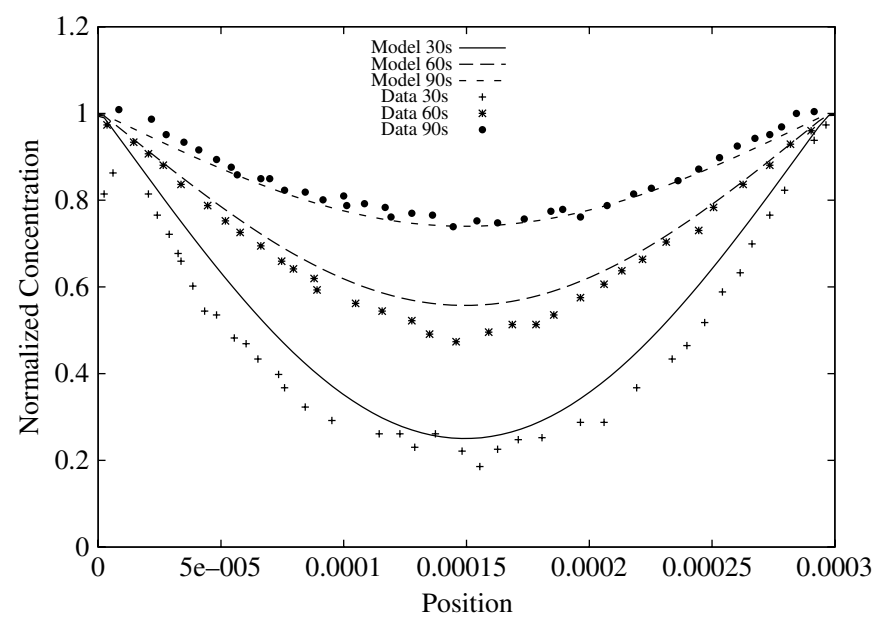

FIGURE 4. Match of the model in (2.2) to the experimental data of Wang et al. [4] for an applied potential of $-0.8 \mathrm{~V}$.

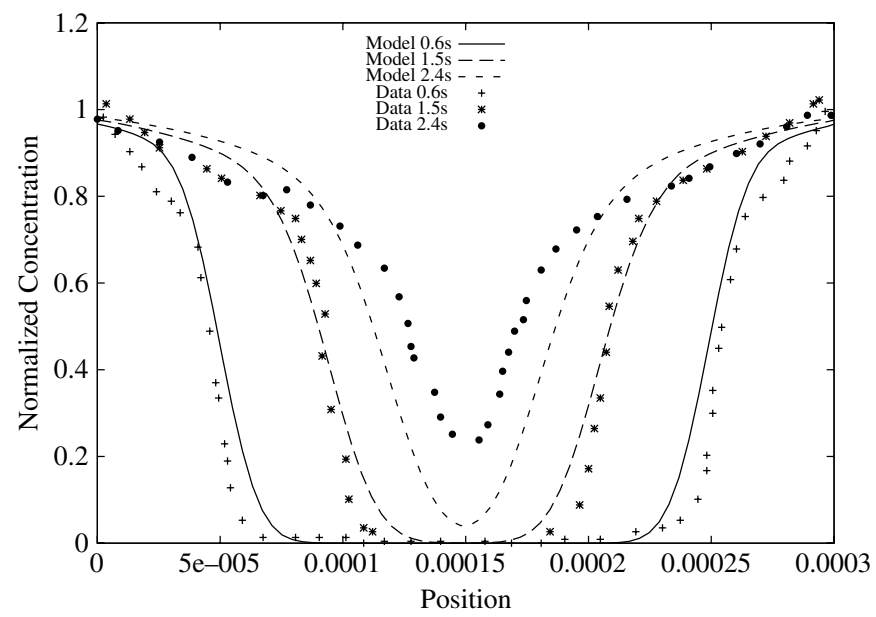

FIGURE 5. Match of the model in (2.2) to the experimental data of Wang et al. [4] for an applied potential of $-1.5 \mathrm{~V}$.

with a Dirichlet condition of a fixed mobile ion concentration assumed,

$$
\left.C^{m}\right|_{x= \pm W_{1}}=C_{0},
$$

where $W_{1}$ is the half-width of the channel of electrolyte $\left(W_{1}>W\right)$.

The system in (2.2) was solved using a $2 \mathrm{D}$ finite-element method on a region $400 \mu \mathrm{m}$ wide, for the cases of low and high applied reducing potentials. The total ion concentration (mobile + immobile) in the polymer is shown in Figures 4 and 5 . The low applied potential case (Figure 4) still shows a good match to the shape 
and velocities of the concentration profiles with the same diffusion coefficient used with (2.1). The case of high applied potential shown in Figure 5 shows improved profile shapes, but the concentration front is still moving too slowly at 2.4 seconds.

The addition to the equations of immobile ions and the transfer term has made a significant difference to the match of the model to concentration profile at the edges. A possible reason why the model underestimates the velocity of the concentration front for larger times is that the model assumes that there is a constant negative charge. However, experiments done with the model to mimic a decrease in the negative charge have not verified this explanation.

\section{A conducting polymer micro-pump}

The behaviour of a conjugated polymer during the redox process where cations and solvent molecules are transported into and out of the polymer results in transient volume changes. Using this behaviour, a micro-actuator can be made from the polymer. This part of the work demonstrates how this property was utilized to design a micro-pump. The goal was to develop a nonmechanical micro-pump that does not require valves and operates at rates of picolitres per second $\left(\mathrm{pL} \mathrm{s}^{-1}\right)$. Such a pump could be used to deliver a continuous supply of a chemical such as a drug or as a continuous addition to a small-scale industrial process.

The idea is to exploit the volume changes that occur during a redox cycle to generate a flow. The pump was designed using a series of micro-actuators which operate out of phase. A finite-element Computational Fluid Dynamics (CFD) model was developed to predict the flow rates of different configurations of the actuators.

There are significant constraints on the pump design imposed from fabrication considerations and the properties of the conjugated polymers. For example:

- the channel dimensions are constrained by fabrication limits-channel heights need to be $10 \mu \mathrm{m}$ or more;

- the actuators need to be greater than $10 \mu \mathrm{m}$ in width and length;

- the volume growth of the actuators results from approximately $1 \mu \mathrm{m}$ growth in the out-of-plane direction - this amount of growth is consistent with experiments done by Smela and Gadegaard [3] and Wu et al. [5];

- the redox reaction requires two seconds for each redox cycle;

- a significant volume of electrolyte is required above the actuators.

Xie et al. [6] have discussed a similar pump design using piezo-electric actuators instead of conjugated polymers. In their design they are able to position the actuators so that the channel closes off, effectively giving them a valve system. This arrangement will be very difficult to achieve using conjugated polymers due to fabrication constraints and the need to maintain sufficient electrolyte above the actuator. Figure 6 shows a schematic of the pump design.

Flow in this micro-pump was calculated using a 2D finite-element CFD model with moving boundaries. Only the fluid flow was explicitly modelled and not ion transport into the polymer. The motion of the polymer micro-actuators was prescribed using 


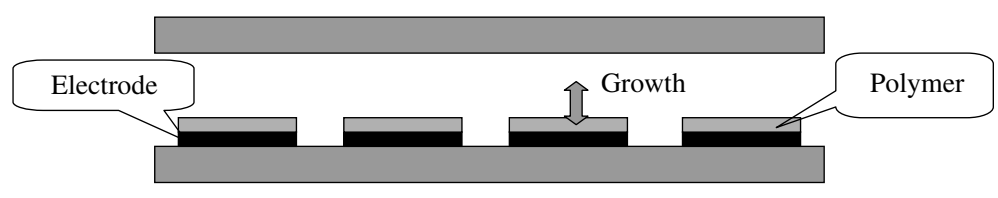

FIGURE 6. Schematic of micro-pump design using conjugated polymer actuators.

a step-function velocity. The actuators were positioned on the bottom face of the channel.

The model equations were the standard Navier-Stokes equations for an incompressible Newtonian fluid, with moving walls. The inlets and outlets were modelled as reservoirs at a prescribed pressure - so that there was no flow if the actuators were still. The aspects of the model that were adjusted were:

- the number of actuators, with two, three, four and five actuators considered;

- the actuator width;

- the channel height - with heights between 2 and $10 \mu \mathrm{m}$ considered, even though heights less than $10 \mu \mathrm{m}$ will be very difficult to fabricate;

- the initial height of the actuators-this corresponds to adjusting the electrode height and the actuator thickness;

- the phase between the actuator velocities.

For the simulations considered here a standardized channel was used. It was $300 \mu \mathrm{m}$ long and $100 \mu \mathrm{m}$ wide. The actuator velocities were set to $\pm 1 \mu \mathrm{m} \mathrm{s}^{-1}$ with a period of two seconds. Simulations were undertaken with varying actuator geometries. The largest flow was obtained for a geometry with three actuators which moved $180^{\circ}$ out of phase, and a channel height of $2 \mu \mathrm{m}$. The length of each actuator was $80 \mu \mathrm{m}$ with a gap of $20 \mu \mathrm{m}$ between the actuators. The sequence is illustrated in Figure 7. Because it takes one second for each actuator to grow, the length of a full cycle of the three actuators is 4 seconds. With a channel height of $2 \mu \mathrm{m}$ and actuators moving $180^{\circ}$ out of phase the flow rate that was achieved was $0.37 \mathrm{pL} \mathrm{s}^{-1}$. As indicated earlier, a $2 \mu \mathrm{m}$ high channel is likely to be difficult to fabricate, so the same model was also run with a $10 \mu \mathrm{m}$ high channel. The flow rate for that channel was $0.09 \mathrm{pL} \mathrm{s}^{-1}$. Further investigations showed that a $10 \mu \mathrm{m}$ high channel with the actuators moving $120^{\circ}$ out of phase gave a flow rate of $0.16 \mathrm{pL} \mathrm{s}^{-1}$. The key difference for this example was the total time for a cycle of $3 \frac{1}{3}$ seconds. These results are summarized in Table 1 . They indicate that it may be possible to achieve picolitre per second flows if a suitable geometry can be fabricated.

The flow rates reported here depend on the actuators growing and displacing fluid. As discussed in the first part of this paper, growth occurs when cations and solvent are transported into the conjugated polymer. For a flow to occur, the volume created in the polymer needs to be greater than the volume occupied by the cations and solvent in the electrolyte. I am currently unaware of any experimental evidence that sheds light on the issue of what flow rates are generated when the polymer grows. In the model, the assumed growth rate is the net growth rate. 

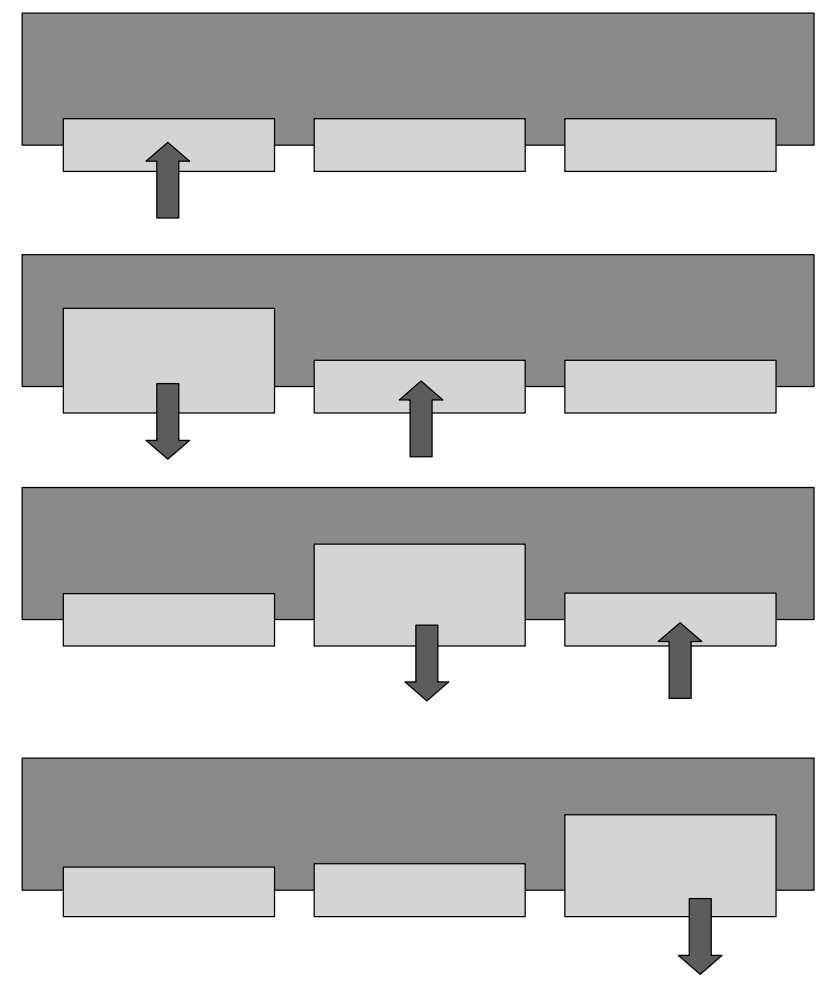

FIGURE 7. Schematic of motion of micro-actuators during a complete cycle in the micro-pump design.

TABLE 1. Summary of flow rates achieved with different configurations.

\begin{tabular}{lc}
\hline Channel conditions & Flow $\left(\mathrm{pL} \mathrm{s}^{-1}\right)$ \\
\hline $2 \mu \mathrm{m}$ high & 0.38 \\
$10 \mu \mathrm{m}$ high & 0.09 \\
$10 \mu \mathrm{m}$ high with step & 0.38 \\
$10 \mu \mathrm{m}$ high, $120^{\circ}$ out of phase & 0.16 \\
$10 \mu \mathrm{m}$ high, actuators top and bottom & 0.65 \\
\hline
\end{tabular}

\section{Conclusions}

A model of ion transport in a conjugated polymer was presented. This model contains diffusive and advective components and extends an earlier model by Wang et al. [4]. The extension distinguishes between mobile and immobile phases of the ions. If a simple phase transfer function is used then the model results match the shapes of the concentration profiles near the boundaries. The model is currently 
unable to explain the increase in the velocity of the concentration front seen as the front moves into the polymer. In fact the model shows this velocity reducing. At the moment the physical process responsible for this behaviour is unknown.

The transport of ions and solvent into conjugated polymers is associated with a volume increase. This change in volume can be exploited to make micro-actuators. Results were presented showing the flow rates that could be developed if such microactuators were used for a micro-pump. For the configuration presented here, such a pump could be expected to generate flows of around $0.2 \mathrm{pL} \mathrm{s}^{-1}$. These flows would be sufficient for a drug delivery at around $0.02 \mu \mathrm{L} /$ day.

\section{References}

[1] W. Plieth, A. Bund, U. Rammelt, S. Neudeck and L. Duc, "The role of ion and solvent transport during the redox process of conducting polymers", Electro. Acta. 51 (2006) 2366-2372.

[2] E. Smela, "Conjugated polymer actuators for biomedical applications", Adv. Mater. 15 (2003) 481-494.

[3] E. Smela and N. Gadegaard, "Volume change in polypyrrole studied by atomic force microscopy", J. Phys. Chem. B 105 (2001) 9395-9405.

[4] X. Wang, B. Shapiro and E. Smela, "Visualizing ion currents in conjugated polymers", Adv. Mater. 16 (2004) 1605-1609.

[5] Y. Wu, G. Alici, G. Spinks and G. Wallace, "Fast trilayer polypyrrole bending actuators for high speed applications", Synth. Met. 156 (2006) 1017-1022.

[6] J. Xie, J. Shih, Q. Lin, B. Yang and Y. Tai, "Dynamic simulation of a peristaltic micropump considering coupled fluid flow and structural motion", J. Micromech. Microeng. 17 (2007) $220-228$. 